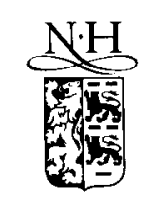

ELSEVIER

\title{
A tree-based adaptive scheme for solution of the equations of gas dynamics and magnetohydrodynamics
}

\author{
Kenneth G. Powell \\ The University of Michigan, Department of Aerospace Engineering, Ann Arbor, MI 48109-2140, USA
}

\begin{abstract}
A solution-adaptive scheme for solving the partial differential equations governing gas dynamics and magnetohydrodynamics is presented. The scheme makes use of a hierarchically structured mesh, in which the cells of the mesh are stored in a quadtree data structure. The scheme is based on a positive linear reconstruction method coupled with an upwind-biased flux function and a multi-stage time-stepping scheme. The upwind flux function for gas dynamics is presented briefly, and the corresponding flux function for the equations governing ideal magnetohydrodynamics is formulated and discussed. Results are presented for problems in gas dynamics and magnetohydrodynamics.
\end{abstract}

Key words: Adaptation; MHD; CFD; Unstructured; Upwind schemes

\section{Introduction}

In order to compute complex flows, researchers in computational fluid dynamics have found it necessary to build an increasing amount of sophistication into their codes. While rapid advances in the capacity of computers have drastically improved the quality of solutions that can be obtained, the increased sophistication of algorithms has had at least as big an effect.

One particular approach to making wise use of computational resources is that of allowing the mesh to adapt to the solution in some way, so that high-gradient regions are not under-resolved, and low-gradient regions are not over-resolved. A rather unfortunate side-effect of implementing this approach is that the mesh-generation algorithm and the flow-solution algorithm become inextricably coupled. This side-effect is one of the primary reasons that adaptive-mesh algorithms are inherently more difficult to implement than fixed-mesh schemes. A second difficulty is that, to facilitate mesh adaptation, more sophisticated data structures than the simple array indexing of structured grids must be used. Connectivity information must be explicitly kept track of, and modified as the mesh is adapted. One final difficulty inherent in the use of adaptively-refined meshes is that large area differences between neighboring cells typically occur. This puts an onus on the flow-solver algorithm, that accuracy can be maintained 
despite the irregularity of the mesh. Recent work in MUSCL schemes for unstructured meshes has shown that this difficulty can be overcome $[1,2]$.

Given the ability to achieve accurate solutions on an irregular mesh, some restrictions on the mesh-generation technique can be relaxed. Also, the use of adaptive refinement ensures a good final mesh, even starting from an less-than-superior initial mesh. Recent work on Cartesianbased solution methods for the Euler equations makes use of these facts [3,8].

The MUSCL approach is very modular-it is made up of a reconstruction scheme, a Riemann-solution-based flux function, a quadrature scheme to integrate the fluxes into a cell of the mesh, and a temporal integration scheme. This modular approach allows Riemann solvers for various problems to be inserted into the same mesh-generation/adaptation framework, so that problems with different physics can be solved by very similar codes.

In the following sections, a mesh-generation, mesh-adaptation, and flow-solution scheme for the equations governing ideal gas dynamics and ideal magnetohydrodynamics is presented. It makes use of Cartesian mesh technology, the MUSCL approach, and adaptive refinement. Results are presented for a variety of cases, demonstrating the power and generality of the approach.

\section{Mesh generation}

\subsection{Data structure}

The data structure used in this work is a cell-based tree. A single cell which encompasses the entire flow domain serves as the root of the tree. A given cell in the tree can be refined, spawning four children cells at the next level of the tree. Some or all of these children cells may then spawn their own children, and so on until the final mesh is built. This process is depicted in Fig. 1.

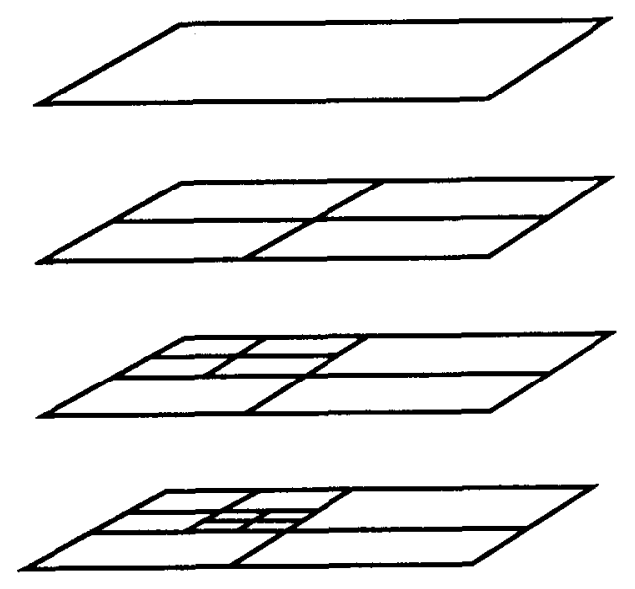

Fig. 1. A cell-based tree data structure. 
This hierarchical structure has several advantages over totally structured or totally unstructured meshes. The tree framework makes it easy to add or delete cells, and is therefore very well suited to solution-adaptive techniques. It is also a natural framework for a multi-grid acceleration procedure, with parent cells combining the residuals of their children to compute coarse-mesh corrections to the fine-mesh states. This data structure may also be used in the mesh-generation procedure, to define an initial mesh that is suitably refined based on geometric features of the problem being solved, as described below.

\subsection{Geometry-based refinement}

The initial-mesh generation is carried out in three steps, depicted in Figs. 2-4. First, a square that encloses the entire flow domain is generated, and enough levels of children cells are spawned that a user-specified cell size is reached. The intersections of this mesh of quadrilaterals with the body (or bodies) in the flow are computed. The mesh produced in this way for a double-ellipse geometry is shown in Fig. 2. At this point, all of the cells which are cut by the body are refined recursively, until a user-specified maximum cell size on the body is reached. The result of this step is shown in Fig. 3. Finally, slopes of neighboring faces on the body are compared, with children cells spawned until a maximum body-curvature threshold is reached. The result is shown in Fig. 4. Obtained with only three simple criteria set by the user, it is an acceptable initial mesh, with the geometry of the problem captured well.

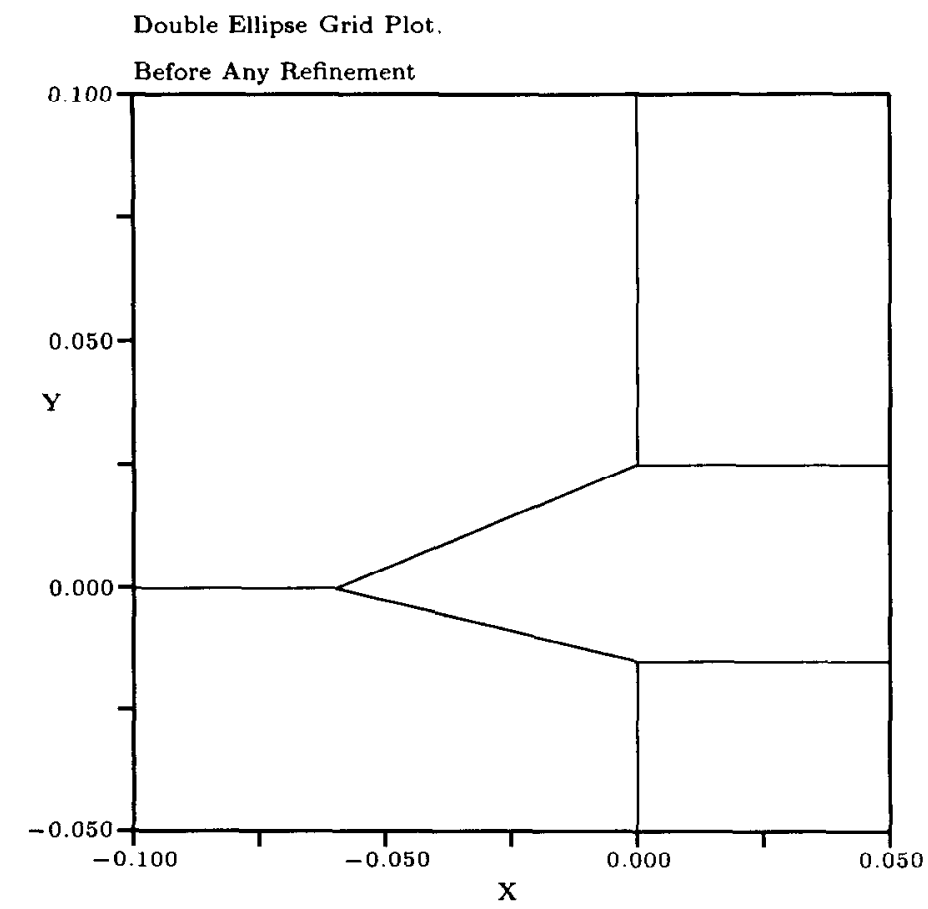

Fig. 2. Detail of double-ellipse mesh after refinement to meet background- $\Delta s$ criterion. 
Double Ellipse Grid Plot.

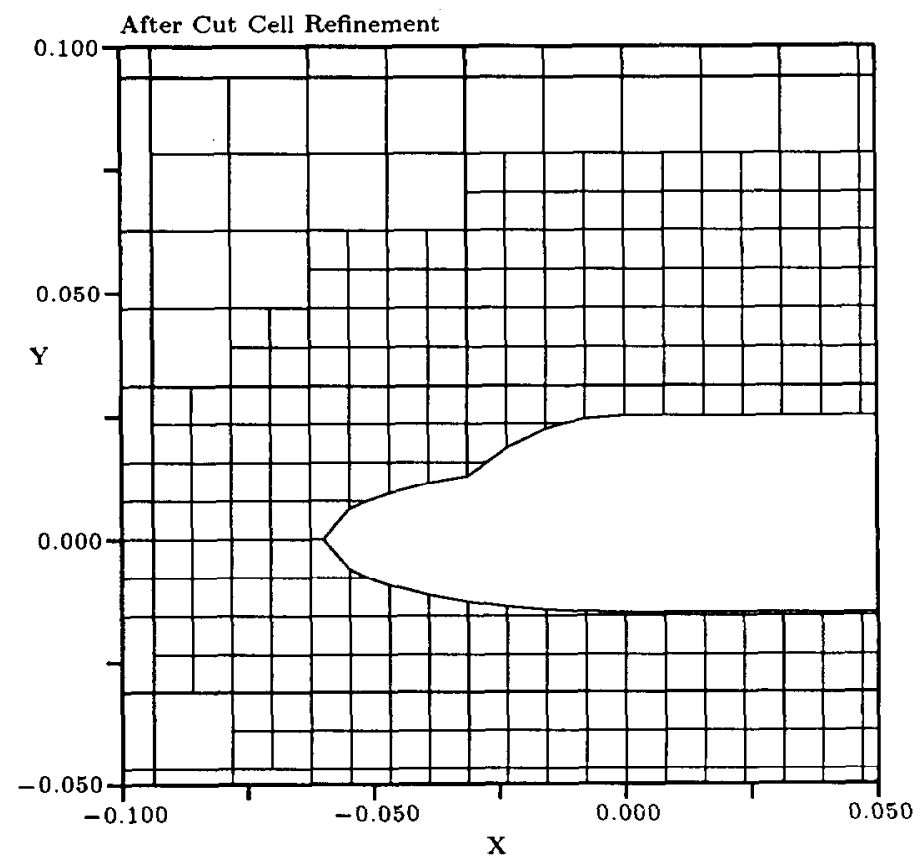

Fig. 3. Detail of double-ellipse mesh after refinement to meet body- $\Delta s$ criterion.

Double Ellipse Grid Plot.

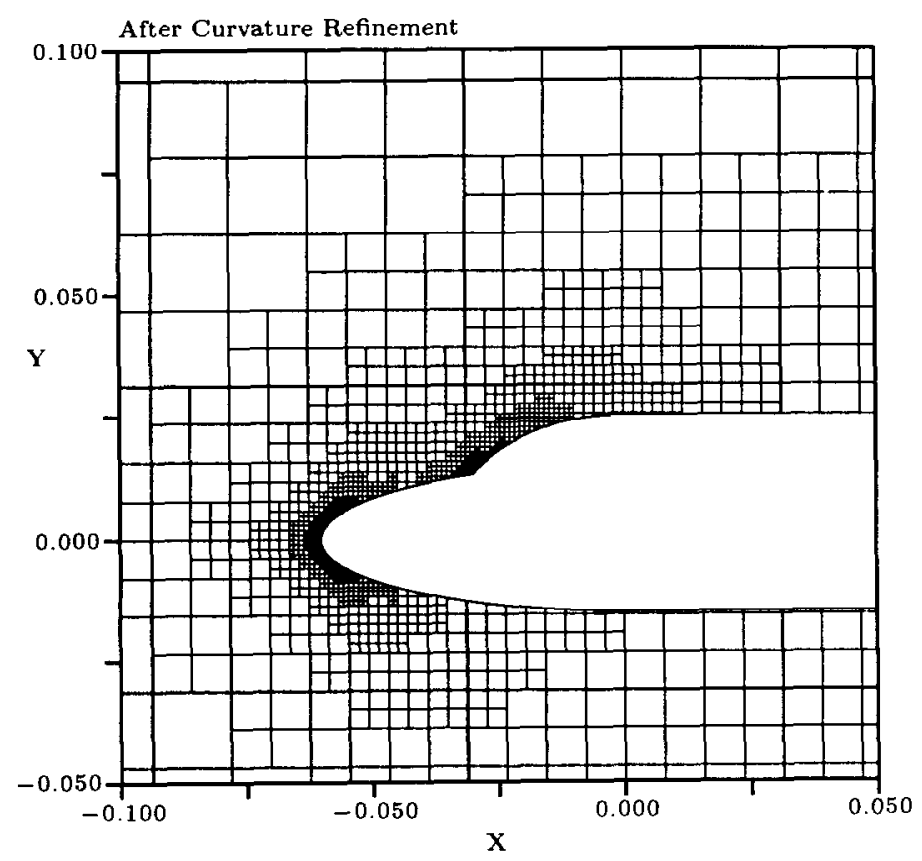

Fig. 4. Detail of double-ellipse mesh after refinement to meet body-curvature criterion. 


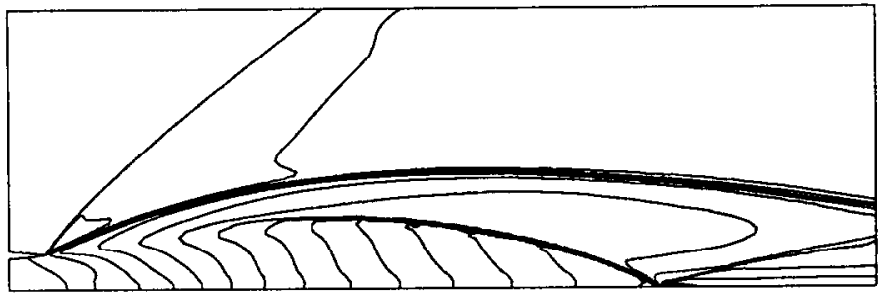

Fig. 5. Under-expanded jet Mach contours.

\subsection{Solution-based refinement}

The mesh may also be adapted based on the flow solution. To do this in an automatic manner, a criterion for determining "important" regions of the flow is necessary, along with thresholds for determining which cells to refine, which to coarsen, and which to leave alone.

Choosing a criterion for determining regions in which adaptation may be necessary is less simple than it might seem; various flow features (e.g. shocks, shears, contacts) manifest themsclves in the solution in various ways. So, for example, a criterion that is well suited for detecting shocks (e.g. pressure gradient) may be poorly suited for detecting shears. One potential solution to this dilemma is to devise a "catch-all" criterion, that detects as many features as possible. This, however, does not address another potential problem-that of flow features of disparate strengths. If a single catch-all criterion is used for a flow with, for example, a strong shear and a weak shock, the shock might not be detected. So, for complex flows, separate criteria, each of which detects one type of feature well, may be better. An example of this is shown in Figs. 5-8. The Mach contours for an under-expanded axisymmetric jet are shown in Fig. 5. The jet boundary is a strong shear; relatively weak shocks are present in the flow as well. The result of refining $15 \%$ of the cells in the mesh, based on a criterion that captures both shocks and shears, is shown in Fig. 6. The shear is detected, but portions of the shocks are missed. The mesh resulting from refining $10 \%$ of the cells, based on a criterion that is tailored to capturing shears is shown in Fig. 7; only the shear is detected. The combination of this shear criterion with an analogous criterion that detects regions of compressibility, such that the two together yield a refinement of $15 \%$ of the cells, the same number of cells as with the

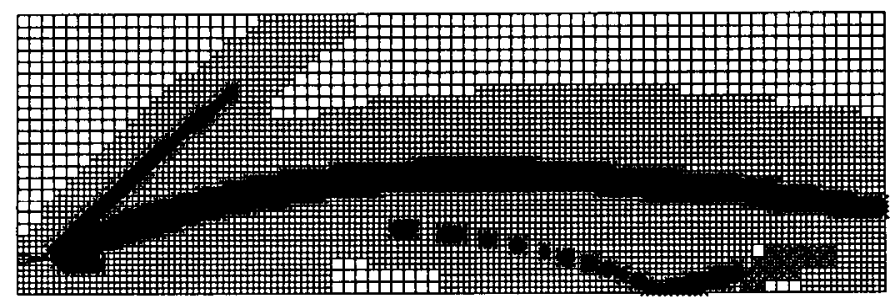

Fig. 6. Under-expanded jet mesh-catch-all criterion. 


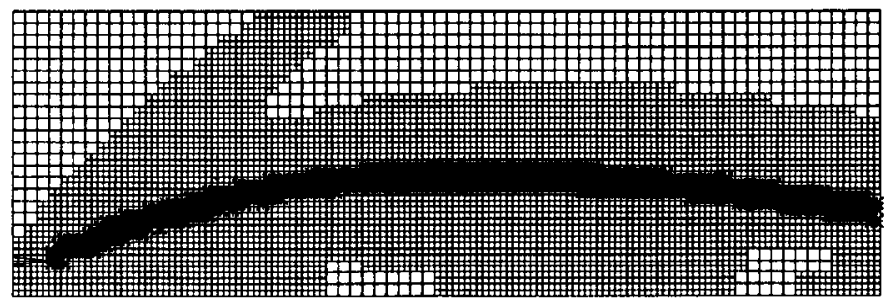

Fig. 7. Under-expanded jet mesh-velocity-curl criterion.

"catch-all" criterion, gives a very good mesh; the shocks, the shear, and the expansion are all detected, as shown in Fig. 8.

The approach taken in this work is to combine two criteria: one specifically designed to detect regions in which compressibility is important; one specifically designed to detect regions in which rotationality is important. To detect regions in which compressibility is important, a properly-scaled velocity divergence is used; to detect regions in which rotationality is important, a properly-scaled velocity curl is used. The choice of the divergence and curl can be justified based on a discrete multidimensional wave model for the gas dynamic equations, which is itself based on the eigensystem of the equations [9].

The "properly-scaled" caveat that appears in the previous paragraph arises from another problem that can occur in adapting the mesh to a given flow solution: that the detection criterion may cause undue refinement of a feature that is already resolved, at the expense of a region of the flow that needs refinement. An excellent (and sobering) depiction of this problem has been given by Warren et al. [18], along with a remedy. The remedy is a careful choice of the constants of proportionality in the adaptation criteria to bc uscd. First-derivative quantities must be multiplied by a factor $h_{i}^{k}$, where $h_{i}$ is a characteristic length for cell $i$, and the power $k$ is chosen so that, as the mesh is refined to the point that the feature is resolved, the value of the refinement criterion, $\tau$, approaches zero. For refinement criteria based on first derivatives, the requirement is that $k>1$; for criteria based on second derivatives, it is $k>2$. For the work presented here, a value $k=3 / 2$ is used.

These adaptation criteria give an analog measure of the "importance" of various regions of the flow. To use them in a scheme that adaptively refines and coarsens the mesh, thresholds for

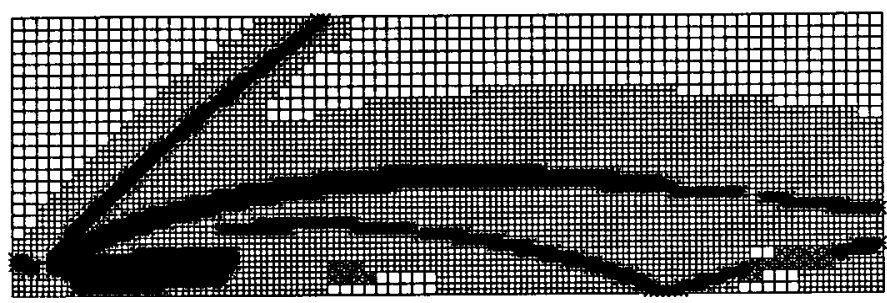

Fig. 8. Under-expanded jet mesh-combined curl/divergence criterion. 
refinement and coarsening must be set for each of the criteria. This is done by examining the distribution function for the criterion $\tau$ on the mesh [7]. Adaptation may be carried out by:

- refining all cells with a value of $\tau$ greater than $x_{1}$ standard deviations above zero;

- coarsening all cells with a value of $\tau$ less than $x_{2}$ standard deviations above zero;

- leaving all remaining cells alone.

The values of $x_{1}$ and $x_{2}$ may be chosen so that a certain percentage of the cells will be flagged for refinement, and a certain percentage flagged for coarsening. Typically, $x_{1}$ is a number between one and two, and $x_{2}$ is on the order of one tenth. Virtually any numbers within this range work well.

The entire scheme for determination of where to adapt the mesh is thus

$$
\begin{array}{cc}
\tau_{\mathrm{r}}=|\nabla \times \boldsymbol{u}| h_{i}^{3 / 2}, & \sigma_{\mathrm{r}}=\sqrt{\frac{\sum_{i=1}^{n} \tau_{\mathrm{r}_{i}}^{2}}{n},} \\
\tau_{\mathrm{c}}=|\nabla \cdot \boldsymbol{u}| h_{i}^{3 / 2}, & \sigma_{\mathrm{c}}=\sqrt{\frac{\sum_{i=1}^{n} \tau_{\mathrm{c}_{i}}^{2}}{n}},
\end{array}
$$

Double Filipse Grid Plot.

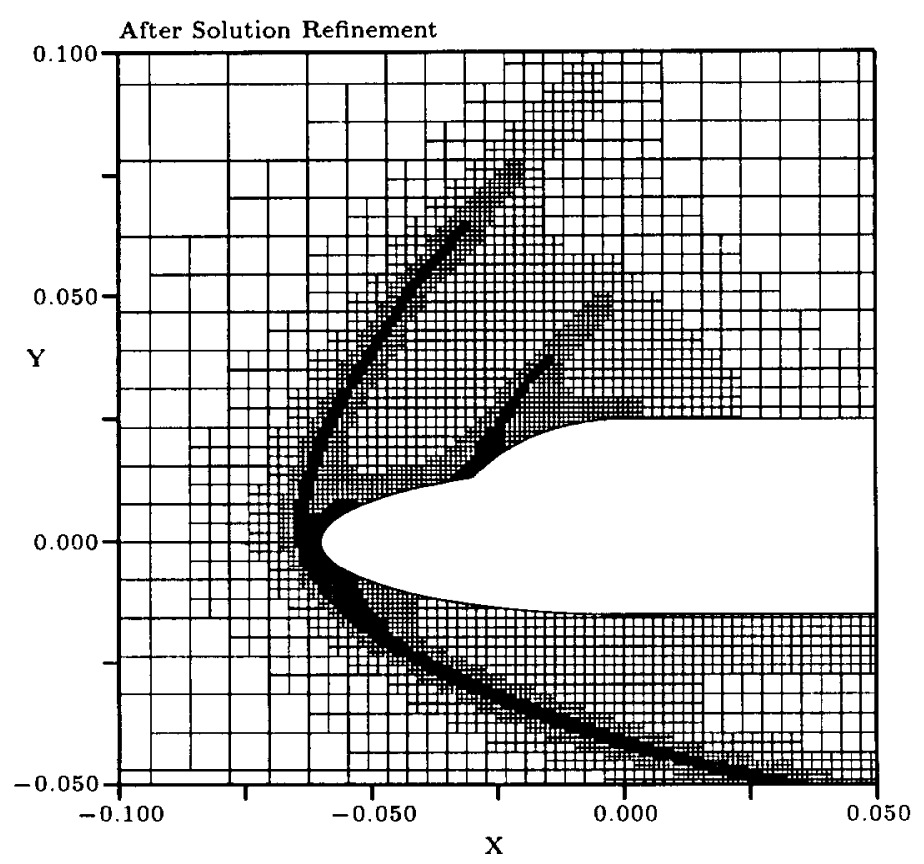

Fig. 9. Detail of double-ellipse mesh after solution-based adaptation. 
flag for refinement if

$$
\left|\tau_{\mathrm{c}}\right|>\sigma_{\mathrm{c}} \text { or }\left|\tau_{\mathrm{r}}\right|>\sigma_{\mathrm{r}}
$$

flag for coarsening if

$$
\left|\tau_{\mathrm{c}}\right|<\frac{1}{10} \sigma_{\mathrm{c}} \text { and }\left|\tau_{\mathrm{r}}\right|<\frac{1}{10} \sigma_{\mathrm{r}}
$$

The result of using this adaptation scheme for the example problem of Section 1 is shown in Fig. 9. The same criteria were used for the gas dynamics and magnetohydrodynamics results in this paper.

\section{Solution scheme}

For a conservation law of the form

$$
\frac{\partial \boldsymbol{U}}{\partial t}+\frac{\partial \boldsymbol{F}}{\partial x}+\frac{\partial \boldsymbol{G}}{\partial y}=0
$$

the finite-volume form can be written as

$$
\frac{\mathrm{d} U}{\mathrm{~d} t}=-\frac{1}{A} \sum_{\text {faces }}(F \Delta y-G \Delta x),
$$

where $A$ is the area of a cell of the mesh, $\Delta x$ and $\Delta y$ are the changes of $x$ and $y$ along a face of the cell (defined so that the integral is carried out in a counter-clockwise sense), and $U$ now represents the local cell average of the state.

Finite-volume techniques can be implemented fairly naturally on unstructured meshes, if care is taken. The most successful approaches have been extensions of Van Leer's MUSCL algorithm [15]. The basic building blocks of a MUSCL-type scheme are:

(1) calculation of the distribution of the flow variables within a cell of the mesh by a reconstruction procedure;

(2) calculation of the fluxes arising from the interaction of neighboring cells, by means of an approximate solution of the Riemann problem;

(3) summation of the fluxes into each cell, and integration in time of the changes to the flow variables.

\subsection{Reconstruction scheme}

To illustrate the reconstruction procedure, a scheme in which the primitive variables are taken to vary linearly over the cells will be described. There are many variations on this scheme: the conserved variables, rather than the primitive variables, can be interpolated, and/or higher-order reconstructions can be done [2].

The vector of primitive variables for the gas dynamics equations is

$$
\boldsymbol{W}=(\rho, u, v, p)^{\mathrm{T}} \text {. }
$$



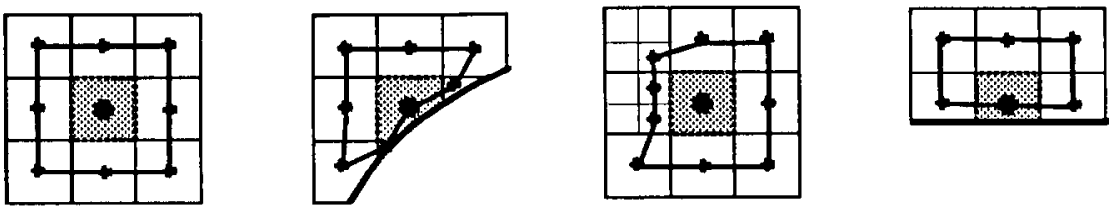

Fig. 10. Some possible paths for gradient calculations on a body-cut Cartesian mesh.

If it is assumed to vary linearly over a cell, then the value of $W$ at any point in the cell is given by

$$
W(x)=\bar{W}+(x-\bar{x}) \cdot \nabla W,
$$

where $\bar{W}$ is the mean value of the primitive variables in the cell, and $\bar{x}$ is the cell centroid. It is clear, then, that an approximation to the gradient of $W$ is necessary to carry out the reconstruction. Since only cell averages of $\boldsymbol{W}$ are given in a finite-volume procedure, this gradient must be calculated from a combination of these averages. One way to do this is by use of Gauss' theorem

$$
\nabla \boldsymbol{W}=\frac{1}{A_{\mathrm{p}}} \oint \boldsymbol{W n} \mathrm{d} s,
$$

where $A_{\mathrm{p}}$ is the area enclosed by the path of integration, and $n$ is the unit outward-pointing normal on the path. Some gradient-integral paths on a body-cut Cartesian mesh are shown in Fig. 10.

An alternate approach to the path-integral approach outlined above is to calculate a least-squares approximation to the gradient in a region [1]. Thus, to get the gradient of $W^{(k)}$, the $k$ th component of the primitive vector $W$, the system to be solved is

$$
\begin{aligned}
& \mathscr{L} \nabla W^{(k)}-f, \\
& \mathscr{L}=\left(\begin{array}{cc}
w_{1} \Delta x_{1} & w_{1} \Delta y_{1} \\
\vdots & \vdots \\
w_{N} \Delta x_{N} & w_{N} \Delta y_{N}
\end{array}\right), \quad f=\left(\begin{array}{c}
w_{1} \Delta W_{1}^{(k)} \\
\vdots \\
w_{N} \Delta W_{N}^{(k)}
\end{array}\right),
\end{aligned}
$$

where

$$
\begin{aligned}
& \Delta x_{i \cdot}=x_{i}-x_{0}, \\
& \Delta y_{i}=y_{i}-y_{0}, \\
& \Delta u_{i}=u_{i}-u_{0},
\end{aligned}
$$

and the points are numbered so that 0 is the origin, as shown in Fig. 11. The $w_{i}$ 's are weight factors, and can be taken to be one, although other values of the weight factors can give gradient approximations that meet certain desirable propertics. This approach, while slightly more expensive computationally than the path-integral approach, is very general, and does not 


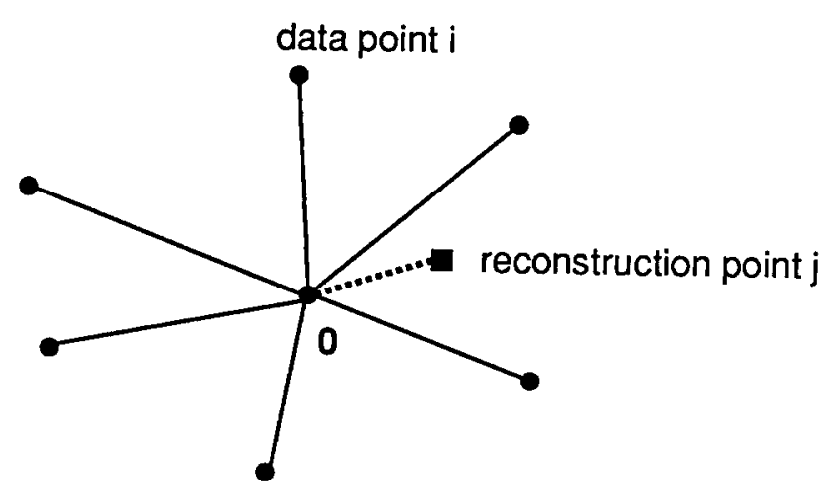

Fig. 11. Numbering of data points.

require any particular ordering of the data used in the gradient calculation. It is the approach taken in the calculations presented in Section 4, with cell areas used as the weighting functions.

Once the gradient has been calculated, values of the primitive variables at the faces may be obtained. However, for the constraint of positivity to be met, the gradient must be limited. That is, the reconstruction formula actually used is

$$
\boldsymbol{W}(\boldsymbol{x})=\bar{W}+\phi(x-\bar{x}) \cdot \nabla \boldsymbol{W},
$$

where $\phi \in[0,1]$ is a limiter. This limiter is calculated so that the values of $\boldsymbol{W}$ reconstructed within the cell are bounded by the data used in the gradient calculation. In regions where

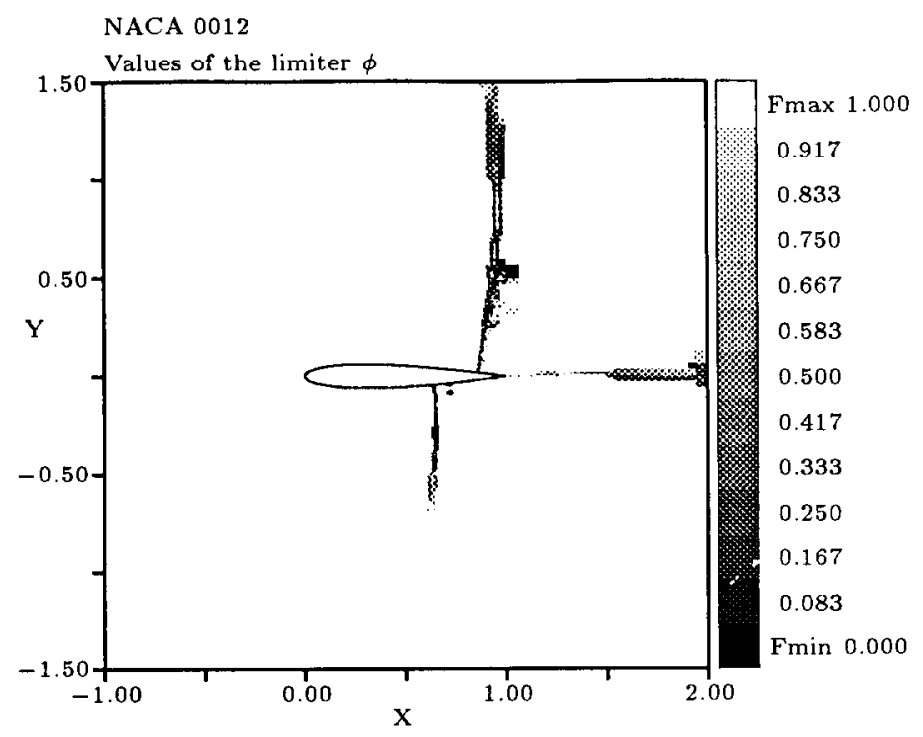

Fig. 12. Values of the limiter $\phi$ for a representative flow calculation. 
$\phi=1$, a piecewise-linear reconstruction is being used; in regions where $\phi=0$, a piecewise-constant reconstruction is being used. One choice for the limiter $\phi$ that assures positivity is

$$
\phi=\min \left\{1, \min _{k}\left(\frac{\left|\bar{W}^{(k)}-\max _{\text {neighbors }}\left(W^{(k)}\right)\right|}{\left|\bar{W}^{(k)}-\max _{\text {cell }}\left(W^{(k)}\right)\right|}\right), \min _{k}\left(\frac{\left|\bar{W}^{(k)}-\min _{\text {neighbors }}\left(W^{(k)}\right)\right|}{\left|\bar{W}^{(k)}-\min _{\text {cell }}\left(W^{(k)}\right)\right|}\right)\right\} .
$$

In the above, $\bar{W}^{(k)}$ denotes the cell-averaged value of the $k$ th component of the vector of primitive variables, and the $\max _{\text {cell }}$ and $\min _{\text {cell }}$ quantities are determined by using the non-limited reconstruction (i.e. Eq. (6)) to determine values at the vertices of the cell.

'Typical values of the limiter are shown in Fig. 12, for a transonic airfoil case. As can be seen, the scheme is higher order $(\phi \approx 1)$ virtually everywhere, reverting to first order $(\phi \approx 0)$ only in the vicinity of the shocks and the wake.

\subsection{Upwind flux functions}

If there were a unique value of $U$ at an interface between two neighboring cells, the flux through a face of length $(\Delta x, \Delta y)$ would be given by

$$
\boldsymbol{\Phi}(\boldsymbol{U})=\boldsymbol{F}(\boldsymbol{U}) \Delta y-\boldsymbol{G}(\boldsymbol{U}) \Delta x .
$$

In the MUSCL approach, however, there are two states associated with an interface, and the flux through the interface is a function of the values of the state variables to the "left" and "right" of the face, as calculated from the reconstruction step in the two cells.

The simplest flux function,

$$
\boldsymbol{\Phi}\left(U_{\mathrm{L}}, U_{\mathrm{R}}\right)=\frac{1}{2}\left[\boldsymbol{\Phi}\left(U_{\mathrm{L}}\right)+\boldsymbol{\Phi}\left(U_{\mathrm{R}}\right)\right]
$$

yields a central-differencing scheme, which is unstable without the addition of some dissipation terms. The dissipation necessary to yield a stable scheme can be introduced in the flux function in a number of ways. The approach taken here is to use Roe's approximate Riemann solver, which is based on the eigensystem of the Jacobian matrix

$$
A=\frac{\partial(F \Delta y-G \Delta x)}{\partial U}
$$

The corresponding flux function is

$$
\boldsymbol{\Phi}\left(\boldsymbol{U}_{\mathrm{L}}, \boldsymbol{U}_{\mathrm{R}}\right)=\frac{1}{2}\left[\boldsymbol{\Phi}\left(\boldsymbol{U}_{\mathrm{L}}\right)+\boldsymbol{\Phi}\left(\boldsymbol{U}_{\mathrm{R}}\right)\right]-\frac{1}{2} \sum_{k=1}^{n_{\text {waves }}}\left|\hat{a}_{k}\right|^{*} \Delta V_{k} \hat{R}_{k},
$$

where the $a_{k}$ 's, $\Delta V_{k}$ 's, and $\hat{\boldsymbol{R}}_{k}$ 's are the speeds, strengths, and eigenvectors, respectively, associated with the waves of the solution to the Riemann problem [12]. The flux functions for the two sets of governing equations are described below. The gas dynamics flux function is well known, and is due to Roe [12]. It is presented here for completeness, and to help provide a framework for the magnetohydrodynamics flux function. The MHD flux function differs from that of Brio and Wu [4] and that of Zachary and Colella [19]. 


\subsubsection{Gas dynamics flux function}

The Euler equations in two dimensions represent the conservation of mass, momentum, and energy for an inviscid, compressible fluid. They may be written as

$$
\frac{\partial \boldsymbol{U}}{\partial t}+\frac{\partial \boldsymbol{F}}{\partial x}+\frac{\partial \boldsymbol{G}}{\partial y}=0
$$

where

$$
\begin{aligned}
\boldsymbol{U} & =(\rho, \rho u, \rho v, \rho E)^{\mathrm{T}}, \\
\boldsymbol{F} & =\left(\rho u, \rho u^{2}+p, \rho u v, \rho u H\right)^{\mathrm{T}}, \\
\boldsymbol{G} & =\left(\rho v, \rho u v, \rho v^{2}+p, \rho v H\right)^{\mathrm{T}},
\end{aligned}
$$

and $H=E+p / \rho$. An equation of state of the form

$$
E=E(p, \rho)
$$

is necessary to close the equations; typically the ideal gas relation

$$
\frac{p}{\rho}=(\gamma-1)\left[E-\frac{1}{2}\left(u^{2}+v^{2}\right)\right]
$$

is used.

Derivation of the wave speeds, strengths, and cigcnvectors has been carried out by Roe $[12,13]$, and is by now well known. They are

$$
\begin{aligned}
\hat{\boldsymbol{a}} & =\left(\begin{array}{c}
\hat{u}_{\mathrm{n}}-\hat{c} \\
\hat{u}_{\mathrm{n}} \\
\hat{u}_{\mathrm{n}} \\
\hat{u}_{\mathrm{n}}+\hat{c}
\end{array}\right), \quad \Delta \boldsymbol{V}=\left(\begin{array}{c}
\left(\Delta p-\hat{\rho} \hat{\boldsymbol{c}} \Delta u_{\mathrm{n}}\right) / 2 \hat{c}^{2} \\
\hat{\rho} \Delta u_{\mathrm{t}} / \hat{c} \\
\Delta \rho-\Delta p / \hat{c}^{2} \\
\left(\Delta p+\hat{\rho} \hat{c} \Delta u_{\mathrm{n}}\right) / 2 \hat{c}^{2}
\end{array}\right), \\
\hat{\boldsymbol{R}} & =\left(\begin{array}{cccc}
1 & 0 & 1 & 1 \\
\hat{u}-\hat{c} \Delta y / \Delta s & \hat{c} \Delta x / \Delta s & \hat{u} & \hat{u}+\hat{c} \Delta y / \Delta s \\
\hat{v}+\hat{c} \Delta x / \Delta s & \hat{c} \Delta y / \Delta s & \hat{v} & \hat{v}-\hat{c} \Delta x / \Delta s \\
H-\hat{u}_{\mathrm{n}} \hat{c} & \hat{u}_{\mathrm{t}} \hat{c} & \frac{1}{2}\left(\hat{u}^{2}+\hat{v}^{2}\right) & H+\hat{u}_{\mathrm{n}} \hat{c}
\end{array}\right),
\end{aligned}
$$

where the components of velocity normal and tangential to the interface,

$$
\begin{aligned}
& u_{\mathrm{n}}=\frac{(u \Delta y-v \Delta x)}{\Delta s} \\
& u_{\mathrm{t}}=\frac{(u \Delta x+v \Delta y)}{\Delta s}
\end{aligned}
$$


are used. The wave speeds and eigenvectors are evaluated at the so-called "Roe-average" state, given by

$$
\begin{aligned}
& \hat{\rho}=\sqrt{\rho_{\mathrm{L}} \rho_{\mathrm{R}}}, \\
& \hat{u}=\frac{\sqrt{\rho_{\mathrm{I}}} u_{\mathrm{L}}+\sqrt{\rho_{\mathrm{R}}} u_{\mathrm{R}}}{\sqrt{\rho_{\mathrm{L}}}+\sqrt{\rho_{\mathrm{R}}}}, \\
& \hat{v}=\frac{\sqrt{\rho_{\mathrm{L}}} v_{\mathrm{L}}+\sqrt{\rho_{\mathrm{R}}} v_{\mathrm{R}}}{\sqrt{\rho_{\mathrm{L}}}+\sqrt{\rho_{\mathrm{R}}}}, \\
& \hat{H}=\frac{\sqrt{\rho_{\mathrm{L}}} H_{\mathrm{L}}+\sqrt{\rho_{\mathrm{R}}} H_{\mathrm{R}}}{\sqrt{\rho_{\mathrm{L}}}+\sqrt{\rho_{\mathrm{R}}}},
\end{aligned}
$$

where $\hat{c}, \hat{u}_{\mathrm{n}}$, and $\hat{u}_{\mathrm{t}}$ are calculated directly from $\hat{\rho}, \hat{u}, \hat{v}$, and $\hat{H}$. Use of the Roe-average state assures ccrtain propertics of the numcrical scheme, including the capturing of grid-aligned shocks and contact discontinuities in a single cell [12].

To prevent expansion shocks, an entropy fix is imposed [16]. A smoothed value, $\left|\hat{a}^{(k)}\right| *$, is defined to replace $\left|\hat{a}^{(k)}\right|$ for the two acoustic waves $(k=1$ and $k=4)$. For those two waves,

$$
\begin{aligned}
& \left|\hat{a}^{(k)}\right|^{*}= \begin{cases}\left|\hat{a}^{(k)}\right|, & \left|\hat{a}^{(k)}\right| \geqslant \frac{1}{2} \delta a^{(k)}, \\
\frac{\left(\hat{a}^{(k)}\right)^{2}}{\delta a^{(k)}}+\frac{1}{4} \delta a^{(k)}, & \left|\hat{a}^{(k)}\right| \leqslant \frac{1}{2} \delta a^{(k)},\end{cases} \\
& \delta a^{(k)}=\max \left(4 \Delta a^{(k)}, 0\right), \quad \Delta a^{(k)}=a_{\mathrm{R}}^{(k)}-a_{\mathrm{L}}^{(k)} .
\end{aligned}
$$

\subsubsection{Magnetohydrodynamics flux function}

The Riemann problem for ideal MHD is a good deal more complicated than that of gas dynamics, and has only recently been used to construct flux functions for upwind schemes $[4,19]$. The equations of ideal MHD arise from writing the equations of motion for a conducting fluid, neglecting the effects of viscosity, heat conduction, resistivity, displacement current, and electrostatic forces. The resulting equations may be written as

$$
\frac{\partial}{\partial t}\left\{\begin{array}{c}
\rho \\
\rho \boldsymbol{u} \\
\boldsymbol{B} \\
E
\end{array}\right\}+\nabla \cdot\left\{\begin{array}{c}
\rho \boldsymbol{u} \\
\rho u \boldsymbol{u}+\boldsymbol{I}\left(\rho+\frac{1}{2} \boldsymbol{B} \cdot \boldsymbol{B}\right)-\boldsymbol{B B} \\
\boldsymbol{u B}-\boldsymbol{B} \boldsymbol{u} \\
(E+p+B \cdot B) \boldsymbol{u}-\boldsymbol{B}(\boldsymbol{B} \cdot \boldsymbol{u})
\end{array}\right\}=0
$$

where $\boldsymbol{B}$ represents the magnetic field, and the total energy is given by

$$
E=\frac{p}{\gamma-1}+\frac{1}{2} \rho \boldsymbol{u} \cdot \boldsymbol{u}+\frac{1}{2} \boldsymbol{B} \cdot \boldsymbol{B} .
$$


The vector of $x$-direction fluxes is thus

$$
\begin{array}{r}
\boldsymbol{F}=\left\{\rho u, \rho u^{2}+p+\frac{1}{2} \boldsymbol{B} \cdot \boldsymbol{B}-B_{x}^{2}, \rho u v-B_{x} B_{y}, \rho u w-B_{x} B_{z}, 0,\right. \\
\left.u B_{y}-v B_{x}, u B_{z}-w B_{x},\left(E+p+\frac{1}{2} \boldsymbol{B} \cdot \boldsymbol{B}\right) u-B_{x} u \cdot \boldsymbol{B}\right\}^{\mathrm{T}},
\end{array}
$$

and the rows of the corresponding Jacobian matrix are

$$
\text { Row } 1=[0,1,0,0,0,0,0,] \text {, }
$$

Row $2=\left[\frac{1}{2}(\gamma-1) \boldsymbol{u} \cdot \boldsymbol{u}-u^{2},-(\gamma-3) u,-(\gamma-1) v,-(\gamma-1) w\right.$,

$$
\left.-\gamma B_{x},-(\gamma-2) B_{y},-(\gamma-2) B_{z}, \gamma-1\right]
$$

Row $3=\left[-u v, v, u, 0,-B_{y},-B_{x}, 0,0\right]$,

Row $4=\left[-u w, w, 0, u,-B_{z}, 0,-B_{x}, 0\right]$,

Row $5=[0,0,0,0,0,0,0,0]$,

Row $6=\left[\frac{v B_{x}-u B_{y}}{\rho}, \frac{B_{y}}{\rho},-\frac{B_{x}}{\rho}, 0,-v, u, 0,0\right]$,

$$
\text { Row } 7=\left[\frac{w B_{x}-u B_{z}}{\rho}, \frac{B_{z}}{\rho}, 0,-\frac{B_{x}}{\rho},-w, 0, u, 0\right] \text {, }
$$

$$
\begin{aligned}
\text { Row } 8=\left[A_{1},\right. & A_{2},-(\gamma-1) u v-\frac{B_{x} B_{y}}{\rho},-(\gamma-1) u w-\frac{B_{x} B_{z}}{\rho}, \\
& -\left(\gamma u B_{x}+v B_{y}+w B_{z}\right),-(\gamma-2) u B_{y}-v B_{x}, \\
& \left.-(\gamma-2) u B_{z}-w B_{x}, \gamma u\right],
\end{aligned}
$$

where

$$
\begin{aligned}
& A_{1}=\frac{B_{x}}{\rho}\left(v B_{y}+w B_{z}\right)-\frac{u\left(B_{y}^{2}+B_{z}^{2}\right)}{\rho}+\frac{1}{2}(\gamma-2) u \boldsymbol{u} \cdot \boldsymbol{u}-\frac{\gamma u p}{(\gamma-1) \rho}, \\
& A_{2}=-(\gamma-1) u^{2}+\frac{1}{2} \boldsymbol{u} \cdot \boldsymbol{u}+\frac{B_{y}^{2}+B_{z}^{2}}{\rho}+\frac{\gamma p}{(\gamma-1) \rho} .
\end{aligned}
$$

This Jacobian matrix has eigenvalues

$$
\begin{aligned}
& \lambda_{\text {ent }}=u, \\
& \lambda_{\text {alf }^{ \pm}}=u \pm B_{x} / \sqrt{\rho}, \\
& \lambda_{\text {slow }^{ \pm}}=u \pm c_{\mathrm{s}}, \\
& \lambda_{\text {fast }^{ \pm}}=u \pm c_{\mathrm{f}}, \\
& \lambda_{\text {div }}=0,
\end{aligned}
$$


where

$$
\begin{aligned}
& c_{\mathrm{s}}^{2}=\frac{1}{2}\left[a^{* 2}-\sqrt{a^{* 4}-4 a^{2} B_{x}^{2} / \rho}\right], \\
& c_{\mathrm{f}}^{2}=\frac{1}{2}\left[a^{* 2}+\sqrt{a^{* 4}-4 a^{2} B_{x}^{2} / \rho}\right], \\
& a^{* 2}=(\gamma p+B \cdot B) / \rho, \\
& a^{2}=\gamma p / \rho .
\end{aligned}
$$

As noted by Brio and Wu [4] and by Zachary and Colella [19], the right and left eigenvectors corresponding to the magnetoacoustic and Alfvén waves must be properly scaled to ensure completeness in various degenerate cases $\left(B_{x} \rightarrow 0, B_{y}^{2}+B_{z}^{2} \rightarrow 0, B \rightarrow 0\right)$. This is done by introducing the variables

$$
\begin{array}{ll}
\beta_{y}=\frac{B_{y}}{\sqrt{B_{y}^{2}+B_{z}^{2}}}, & \beta_{z}=\frac{B_{z}}{\sqrt{B_{y}^{2}+B_{z}^{2}}}, \\
\alpha_{\mathrm{s}}=\frac{\sqrt{c_{\mathrm{f}}^{2}-a^{2}}}{\sqrt{c_{\mathrm{f}}^{2}-c_{\mathrm{s}}^{2}}}, & \alpha_{\mathrm{f}}=\frac{\sqrt{\rho c_{\mathrm{f}}^{2}-B_{x}^{2}}}{\sqrt{\rho c_{\mathrm{f}}^{2}-\rho c_{\mathrm{s}}^{2}}},
\end{array}
$$

and expressing the Alfvén and magnetoacoustic waves in terms of them.

The eigenvector corresponding to the entropy wave is

$$
R_{\mathrm{ent}}=\left(1, u, v, w, 0,0,0, \frac{1}{2} \boldsymbol{u} \cdot u\right)^{\mathrm{T}}
$$

The properly-normalized Alfvén wave eigenvectors are

$$
R_{\mathrm{alf} \pm}=\left(0,0, \mp \beta_{z}, \pm \beta_{y}, 0, \frac{\beta_{z}}{\sqrt{\rho}},-\frac{\beta_{y}}{\sqrt{\rho}}, \pm\left(w \beta_{y}-v \beta_{z}\right)\right)^{\mathrm{T}}
$$

The slow magnetoacoustic eigenvectors are

$$
\begin{gathered}
R_{\text {slow } \pm}=\left(\alpha_{\mathrm{s}}, \alpha_{\mathrm{s}}\left(u \pm c_{\mathrm{s}}\right), \alpha_{\mathrm{s}} v \pm \alpha_{\mathrm{f}} a \beta_{y} \operatorname{sgn} B_{x}, \alpha_{\mathrm{s}} w \pm \alpha_{\mathrm{f}} a \beta_{z} \operatorname{sgn} B_{x}, 0,\right. \\
\left.-\frac{\alpha_{\mathrm{f}} a^{2} \beta_{y}}{\sqrt{\rho} c_{\mathrm{f}}},-\frac{\alpha_{\mathrm{f}} a^{2} \beta_{z}}{\sqrt{\rho} c_{\mathrm{f}}}, \frac{1}{2} \alpha_{\mathrm{s}} u \cdot u+\alpha_{\mathrm{s}} h_{\mathrm{s}}^{ \pm}\right)
\end{gathered}
$$

where

$$
\alpha_{\mathrm{s}} h_{\mathrm{s}}^{ \pm}=\alpha_{\mathrm{s}}\left(\frac{c_{\mathrm{s}}^{2}}{\gamma-1} \pm c_{\mathrm{s}} u+\frac{\gamma-2}{\gamma-1}\left(c_{\mathrm{s}}^{2}-a^{2}\right)\right) \pm \alpha_{\mathrm{f}}\left(\beta_{y} v+\beta_{z} w\right) a \operatorname{sgn} B_{x} .
$$

The fast magnetoacoustic eigenvectors are

$$
\begin{gathered}
R_{\text {fast }^{ \pm}}=\left(\alpha_{\mathrm{f}}, \alpha_{\mathrm{f}}\left(u \pm c_{\mathrm{f}}\right), \alpha_{\mathrm{f}} v \mp \alpha_{\mathrm{s}} \frac{B_{x}}{\sqrt{\rho}} \beta_{y}, \alpha_{\mathrm{f}} w \mp \alpha_{\mathrm{s}} \frac{B_{x}}{\sqrt{\rho}} \beta_{z}, 0,\right. \\
\left.\frac{\alpha_{\mathrm{s}} c_{\mathrm{f}} \beta_{y}}{\sqrt{\rho}}, \frac{\alpha_{\mathrm{s}} c_{\mathrm{f}} \beta_{z}}{\sqrt{\rho}}, \frac{1}{2} \alpha_{\mathrm{f}} \boldsymbol{u} \cdot \boldsymbol{u}+\alpha_{\mathrm{f}} h_{\mathrm{f}}^{+}\right)
\end{gathered}
$$


where

$$
\alpha_{\mathrm{f}} h_{\mathrm{f}}^{ \pm}=\alpha_{\mathrm{f}}\left(\frac{c_{\mathrm{f}}^{2}}{\gamma-1} \pm c_{\mathrm{f}} u+\frac{\gamma-2}{\gamma-1}\left(c_{\mathrm{f}}^{2}-a^{2}\right)\right) \mp \alpha_{\mathrm{s}}\left(\beta_{y} v+\beta_{z} w\right) \frac{B_{x}}{\sqrt{\rho}}
$$

It should be noted that the final wave does not contribute to the flux in the usual manner, since the wave speed is zero. It does, however, act to alter the strengths of the other seven waves, in the case that the jump in the normal component of the magnetic field is non-zero across a cell face. In a one-dimensional case, or a case in which the flow is two-dimensional and the magnetic field is aligned with the third dimension, the jump is zero, and so this wave does not affect the other wave strengths. In a flow with a fully three-dimensional magnetic field, however, this effect would need to be included. For the calculation shown in the results section, a two-dimensional flow, with the magnetic field aligned with the $z$-axis, is shown.

The strengths for the other waves can be expressed most simply in terms of the sums and differences of strengths as follows.

$$
\left(\begin{array}{c}
\alpha_{\text {slow }^{+}}-\alpha_{\text {slow }^{-}} \\
\alpha_{\text {fast }^{+}}-\alpha_{\text {fast }^{-}}
\end{array}\right)=\frac{1}{J_{1}}\left[\begin{array}{cc}
\alpha_{\mathrm{s}}\left|B_{x}\right| / \sqrt{\rho} & \alpha_{\mathrm{f}} c_{\mathrm{f}} \operatorname{sgn} B_{x} \\
\alpha_{\mathrm{f}} a & -\alpha_{\mathrm{s}} c_{\mathrm{s}} \operatorname{sgn} B_{x}
\end{array}\right]\left(\begin{array}{l}
\delta_{1} \\
\delta_{2}
\end{array}\right),
$$

where

$$
\begin{aligned}
& J_{1}=\alpha_{\mathrm{s}}^{2} c_{\mathrm{s}} \frac{\left|B_{x}\right|}{\sqrt{\rho}}+\alpha_{\mathrm{f}}^{2} c_{\mathrm{f}} a, \\
& \delta_{1}=\rho \Delta u, \quad \delta_{2}=\beta_{y} \rho \Delta v+\beta_{z} \rho \Delta w \\
& \left(\begin{array}{l}
\alpha_{\text {slow }^{+}}+\alpha_{\text {slow }^{-}} \\
\alpha_{\text {fast }^{+}}+\alpha_{\text {fast }^{-}}
\end{array}\right)=\frac{1}{J_{2}}\left[\begin{array}{cc}
-\alpha_{\mathrm{f}}\left(\frac{\gamma-2}{\gamma-1} a^{2}-c_{\mathrm{f}}^{2}\right) \sqrt{\rho} c_{\mathrm{f}} & -\alpha_{\mathrm{s}} c_{\mathrm{f}}^{2} \\
\alpha_{\mathrm{s}}\left(\frac{\gamma-2}{\gamma-1} a^{2}-c_{\mathrm{s}}^{2}\right) \sqrt{\rho} c_{\mathrm{f}} & -\alpha_{\mathrm{f}} a^{2}
\end{array}\right]\left(\begin{array}{l}
\delta_{3} \\
\delta_{4}
\end{array}\right),
\end{aligned}
$$

where

$$
\begin{aligned}
& J_{2}=\alpha_{\mathrm{s}}^{2} c_{\mathrm{f}}^{2}\left[\frac{\gamma-2}{\gamma-1} a^{2}-c_{2}^{2}\right]+\alpha_{\mathrm{f}}^{2} a^{2}\left[\frac{\gamma-2}{\gamma-1} a^{2}-c_{\mathrm{f}}^{2}\right], \\
& \delta_{3}=\beta_{y} \Delta B_{y}+\beta_{z} \Delta B_{z}, \quad \delta_{4}=\frac{\Delta p}{\gamma-1}+\Delta\left(\frac{1}{2} \boldsymbol{B} \cdot \boldsymbol{B}\right) \\
& \left(\begin{array}{l}
\alpha_{\mathrm{alf}^{+}}-\alpha_{\mathrm{alf}^{-}} \\
\alpha_{\mathrm{alf}^{+}}+\alpha_{\mathrm{alf}^{-}}
\end{array}\right)=\left(\begin{array}{c}
\delta_{5} \\
\delta_{6}
\end{array}\right),
\end{aligned}
$$

where

$$
\delta_{5}=\beta_{y} \rho \Delta w-\beta_{z} \rho \Delta v, \quad \delta_{6}=\sqrt{\rho} \beta_{z} \Delta B_{y}-\sqrt{\rho} \beta_{y} \Delta B_{z} .
$$


For the magnetohydrodynamics Riemann problem, a Roe-average which exhibits Property U $[12,13]$ does not exist, except in the special case $\gamma=2[4]$. For this reason, a simple arithmetic average of the left and right states is used for the interface state at which the eigenvectors and wave speeds are evaluated. In addition, an entropy fix is applied to the four magnetoacoustic waves.

\subsection{Time-stepping scheme}

The time-stepping scheme used is one of the optimally-smoothing multi-stage schems developed by Tai $[14,17]$. The general $m$-stage scheme is defined as

$$
\begin{aligned}
& \boldsymbol{U}^{(0)}=\boldsymbol{U}^{n}, \\
& \boldsymbol{U}^{(k)}=\boldsymbol{U}^{(0)}+\boldsymbol{\alpha}_{k} \Delta t \boldsymbol{\operatorname { R e s }}\left(\boldsymbol{U}^{(k-1)}\right), \quad k=1, \ldots, m, \\
& \boldsymbol{U}^{n+1}=\boldsymbol{U}^{(m)} .
\end{aligned}
$$

Coefficients $\alpha_{k}$ and time stcps can bc chosen for an $m$-stage scheme with a given spatial operator so that high frequency error waves are optimally (in a min-max sense) damped.

Local time-stepping is used, and indeed is necessary. The meshes generated have extremely large differences in area from cell to cell, due to the cut cells. The local time-stepping removes the stiffness due to this area variation, and convergence is virtually as rapid as on an unstretched mesh. A representative convergence history is shown in Fig. 13. The spikes in the convergence history occur when the grid is adapted.

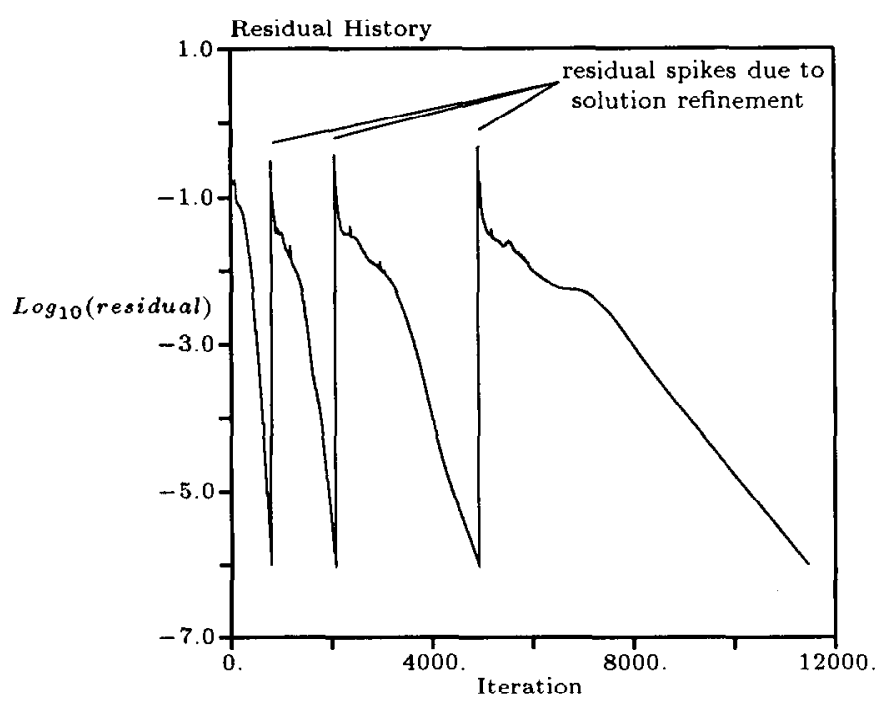

Fig. 13. Representative convergence history. 


\section{Results}

\subsection{Branched duct flow}

Some results for a branched duct flow are shown in Figs. 14-17. This flow is representative of that within the coolant passages of some turbine blades, with a splitter plate and an array of pins. The pins are present primarily to provide blockage. The mesh, shown in Fig. 14, is refined about the pins, and about the two shear layers which emanate from the $90^{\circ}$ corner and the splitter plate. The Mach contours (Figs. 15 and 16) show the compressions and expansions caused by the pins, as well as the wakes behind them. In addition, evidence of a corner eddy and the roll-up of the shear emanating from the edge of the splitter plate can be seen. The contours of total pressure loss, presented in Fig. 17, show evidence of these two vortices, since vortices tend to display a substantial total pressure loss in their cores, even in Euler calculations $[10,11]$. This case demonstrates the ability of the mesh-generation scheme to capture relatively complex geometries automatically. Some care must be taken in interpreting the results, as the inviscid computation obviously cannot model the regions of the flow in which viscosity is very important (e.g. the wakes behing the pins).

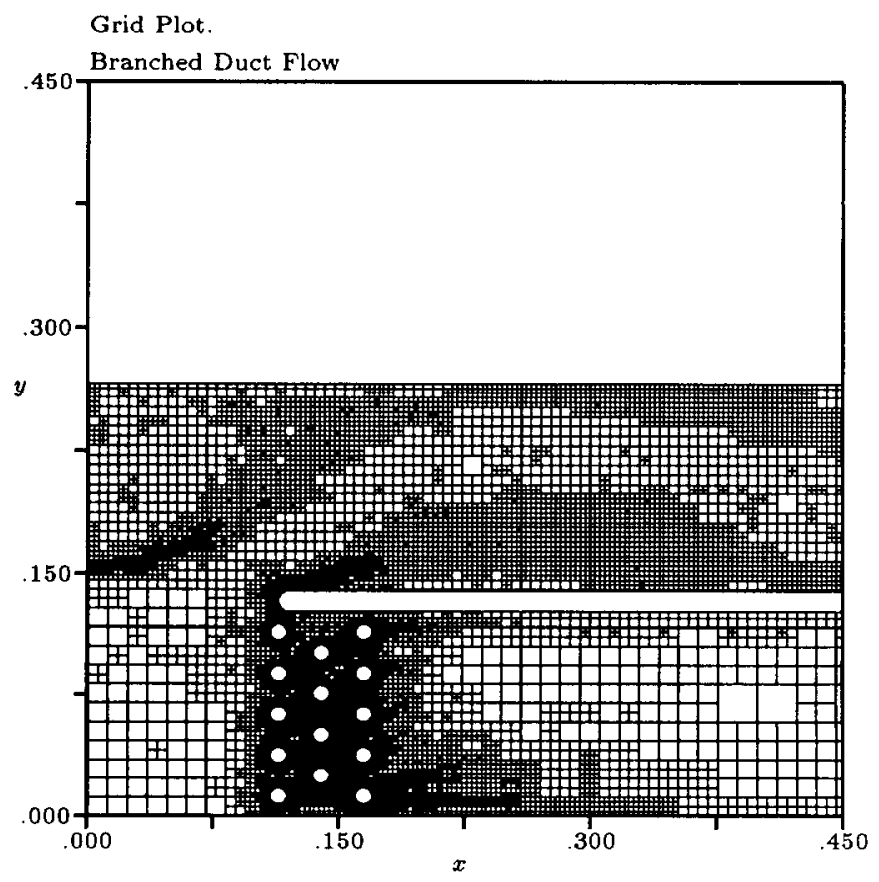

Fig. 14. Grid for branched duct case. 
Mach Number Line Contours.

Branched Duct Flow

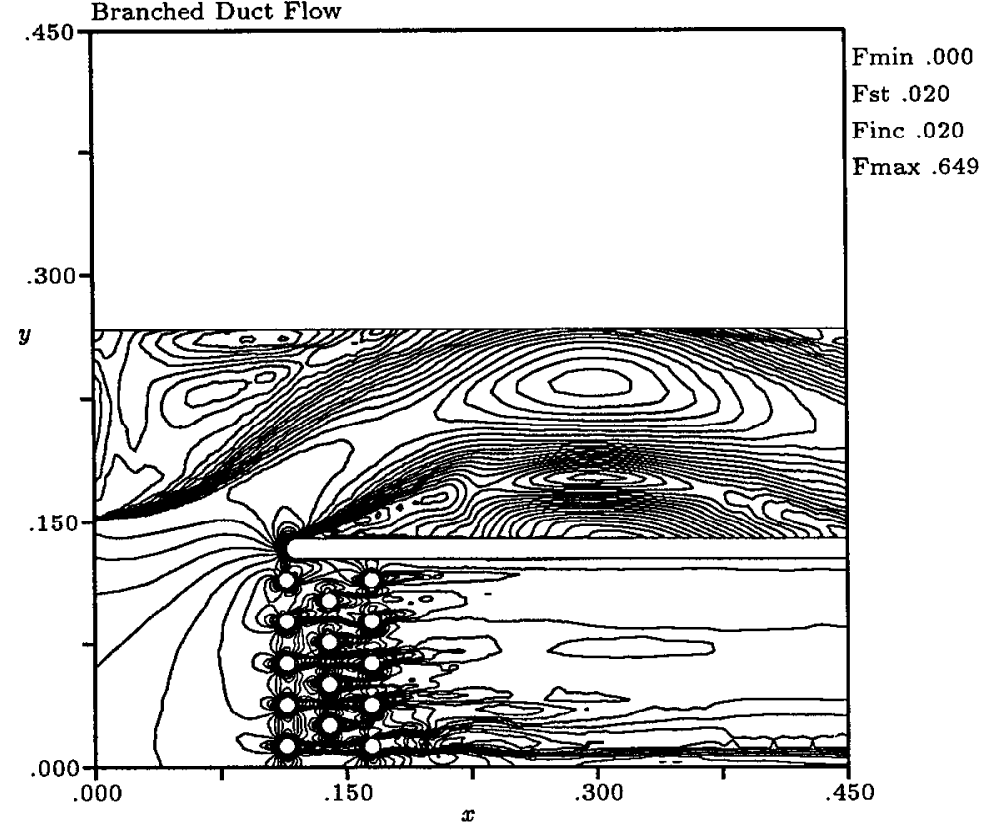

Fig. 15. Mach contours for branched duct case.

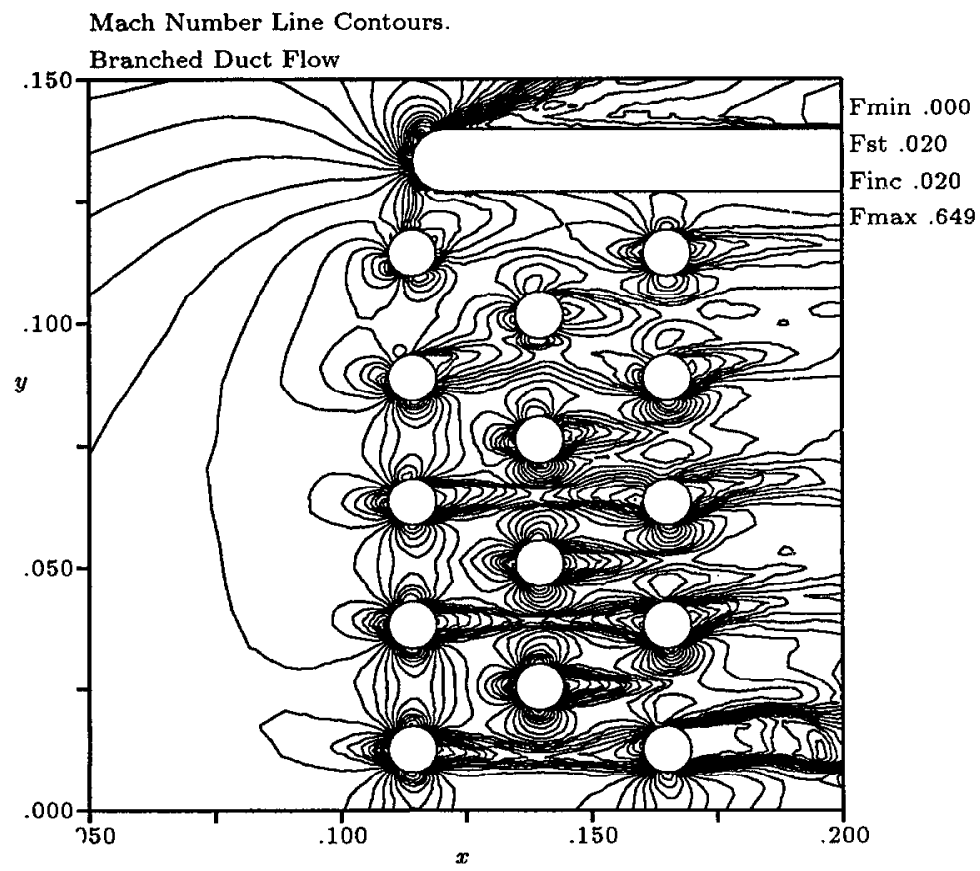

Fig. 16. Mach contours for branched duct case (detail). 


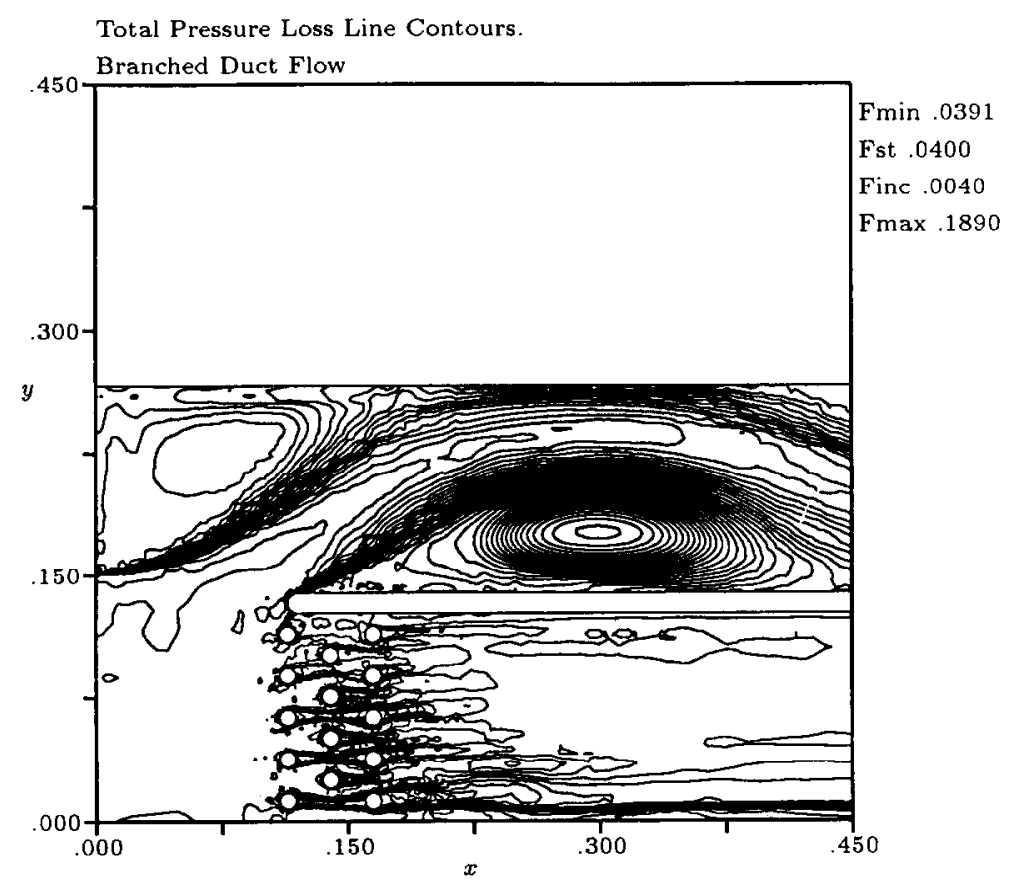

Fig. 17. Total pressure loss contours for branched duct case.

\subsection{Blast wave}

The results of an unsteady calculation representing an axisymmetric blast wave are shown in Figs. 18-25. the initial conditions were a quiescent fluid, with a shock moving at speed $M=1.76$ through the cylindrical tube. The evolution in time of the grid and the contours of density demonstrate the ability of the scheme to capture complex shock-shear systems efficiently and accurately. The shocks are captured across a very few grid points, and relatively weak shocks are detected by the adaptation scheme for refinement. The shear emanating from the triple point at the latest time depicted shows some instability due to the capturing of the shcar with a low order of dissipation.

For this unsteady case, a two-stage time-stepping procedure that is second-order accurate in time was used, and adaptation was done at each time step [5,6].

\subsection{Flow past a conducting cylinder}

Results of the scheme using the magnetohydrodynamic Riemann solver for the flow past a conducting cylinder are shown in Figs. 26 and 27. The incoming acoustic Mach number is 2.0; the incoming Alfvénic Mach number is 3.0, with the magnetic field in the $z$-direction. A bow shock forms in front of the cylinder, and the expansion around the cylinder leads to two tail shocks. There is shock-induced separation of the flow behind the cylinder. Fig. 26 shows the grid for this case; Fig. 27 shows the Alfvénic Mach number $(\rho \boldsymbol{u} \cdot \boldsymbol{u} / \boldsymbol{B} \cdot \boldsymbol{B})$. This flow, while 
Grid Plot.

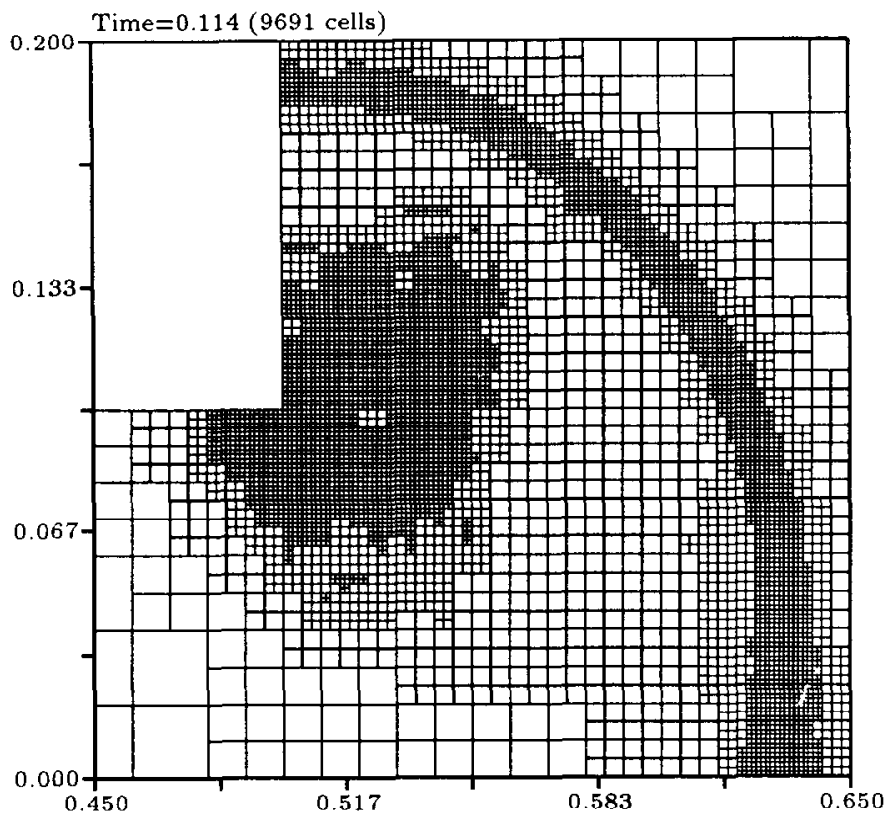

Fig. 18. Grid for blast wave at $t=0.114$.

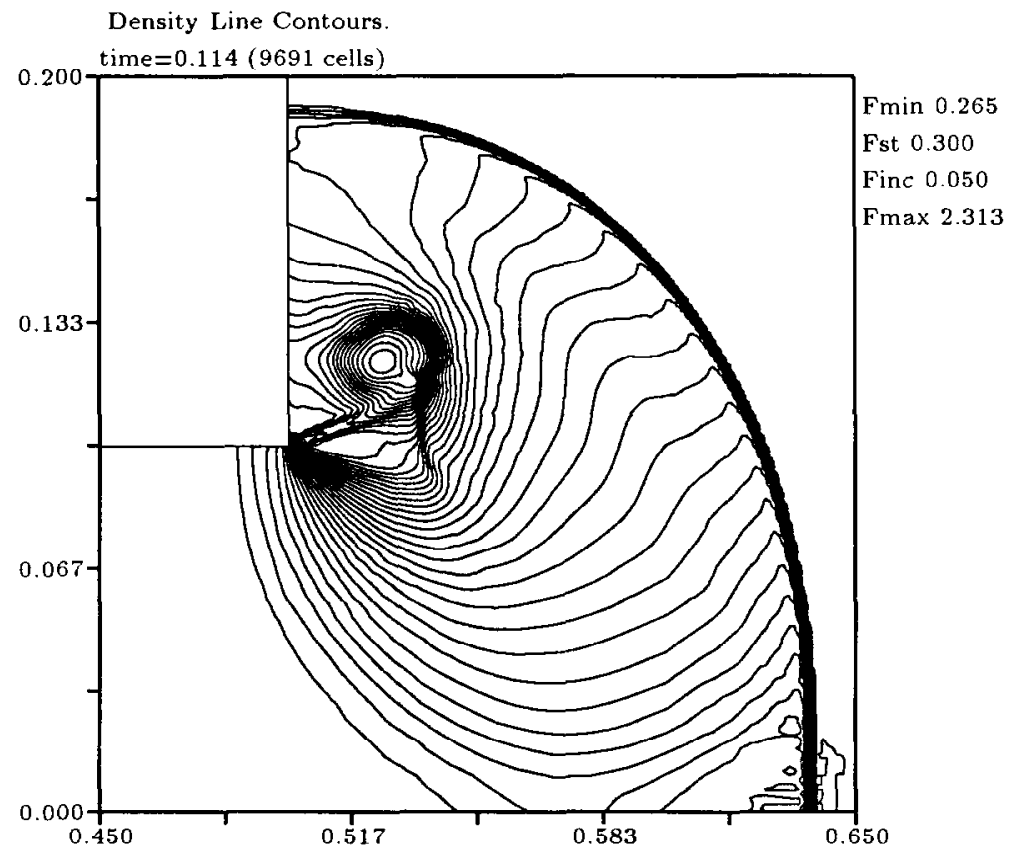

Fig. 19. Density contours for blast wave at $t=0.114$. 
Grid Plot.

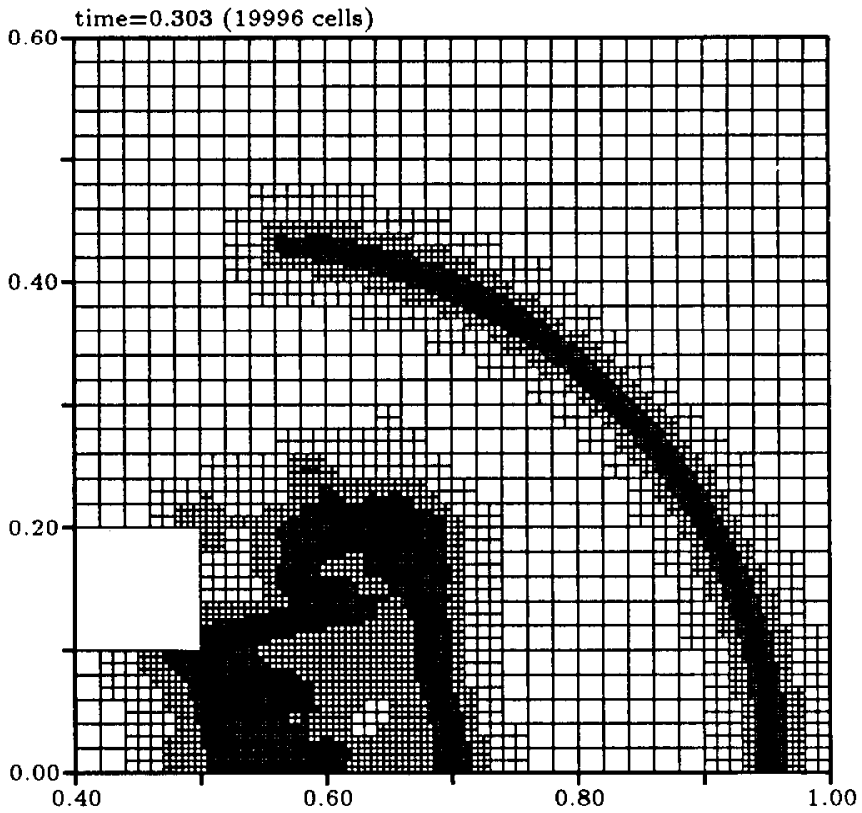

Fig. 20. Grid for blast wave at $t=0.303$.

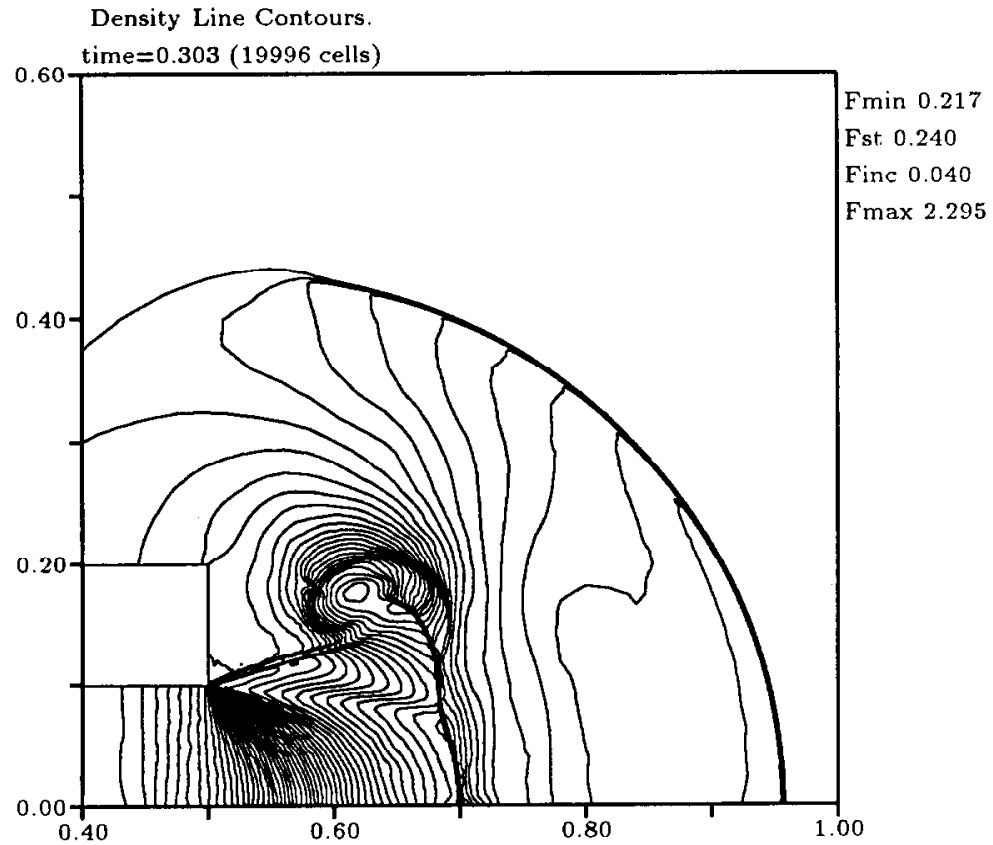

Fig. 21. Density contours for blast wave at $t=0.303$. 
Grid Plot.

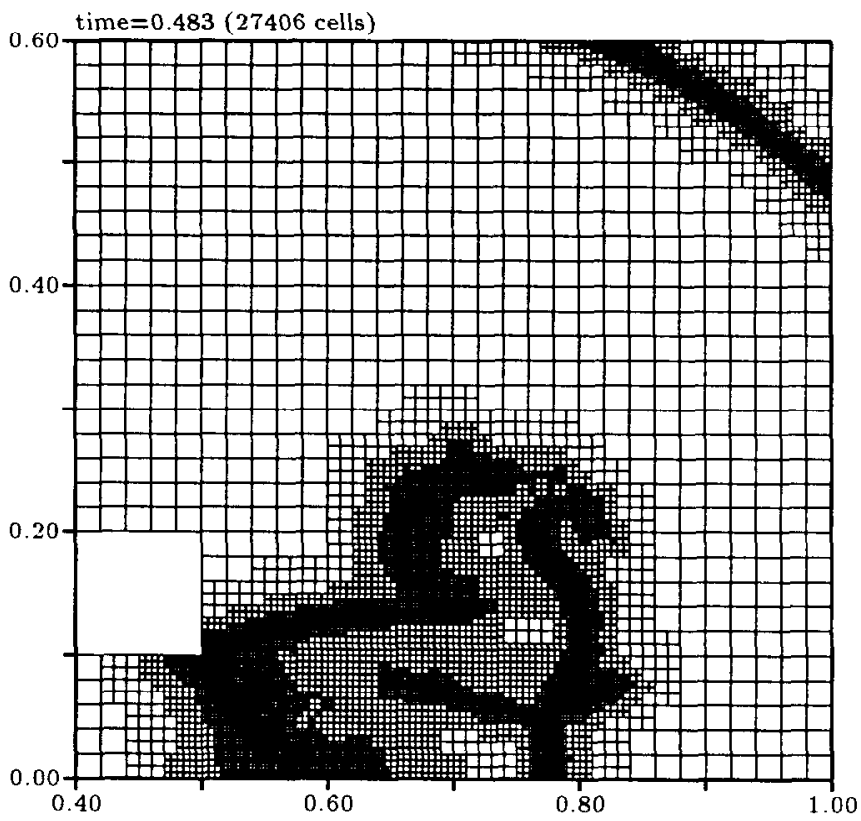

Fig. 22. Grid for blast wave at $t=0.483$.

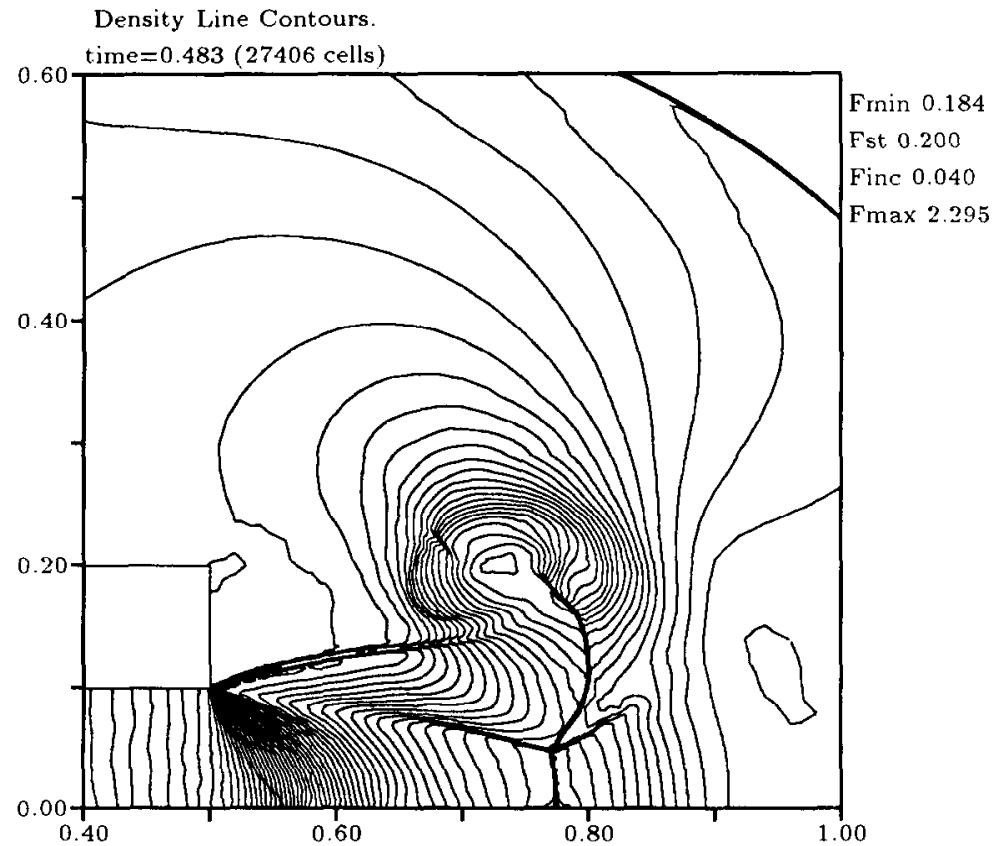

Fig. 23. Density contours for blast wave at $t=0.483$. 
Grid Plot.

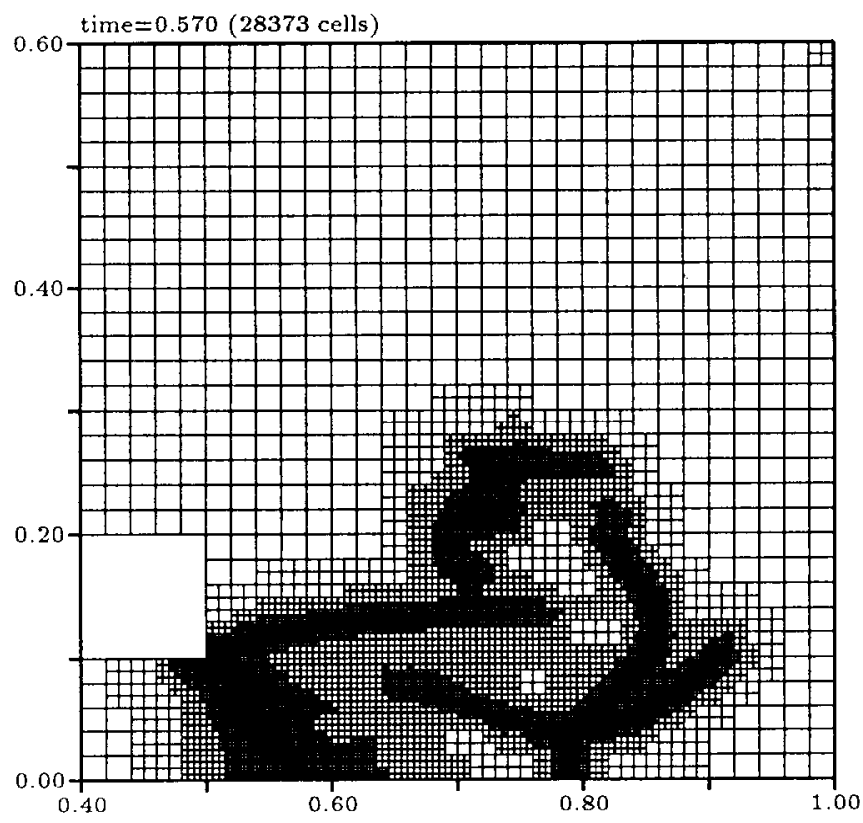

Fig. 24. Grid for blast wave at $t=0.570$.

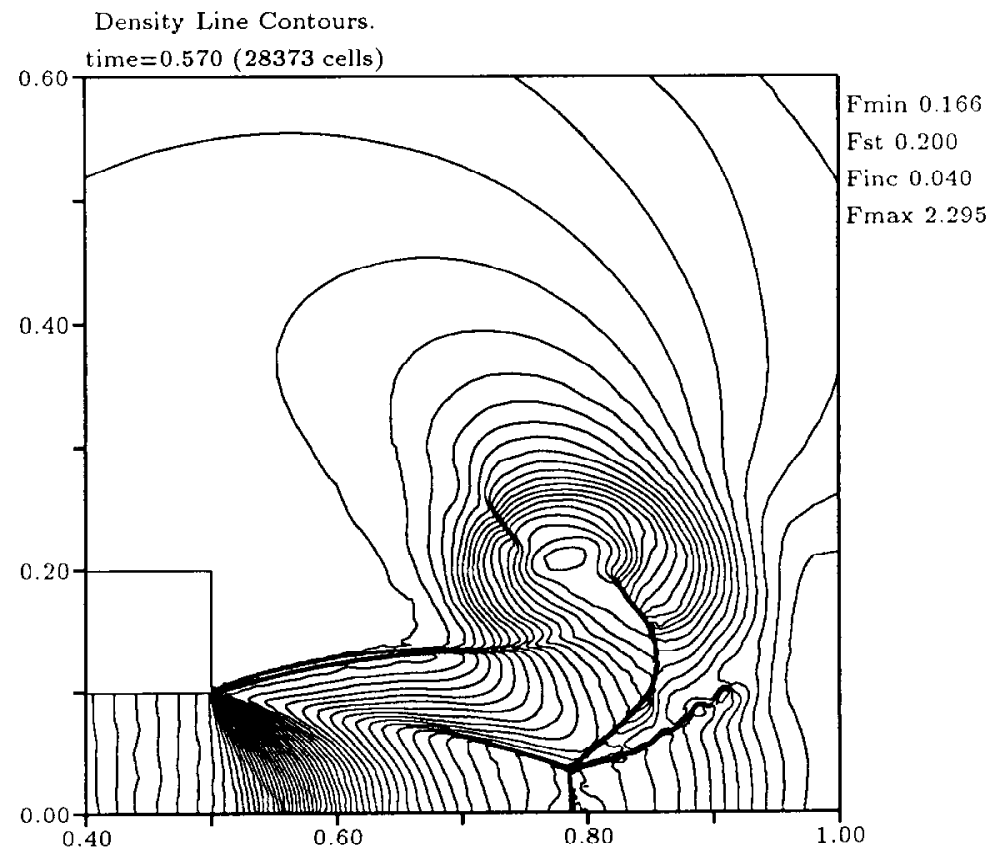

Fig. 25. Density contours for blast wave at $t=0.570$. 


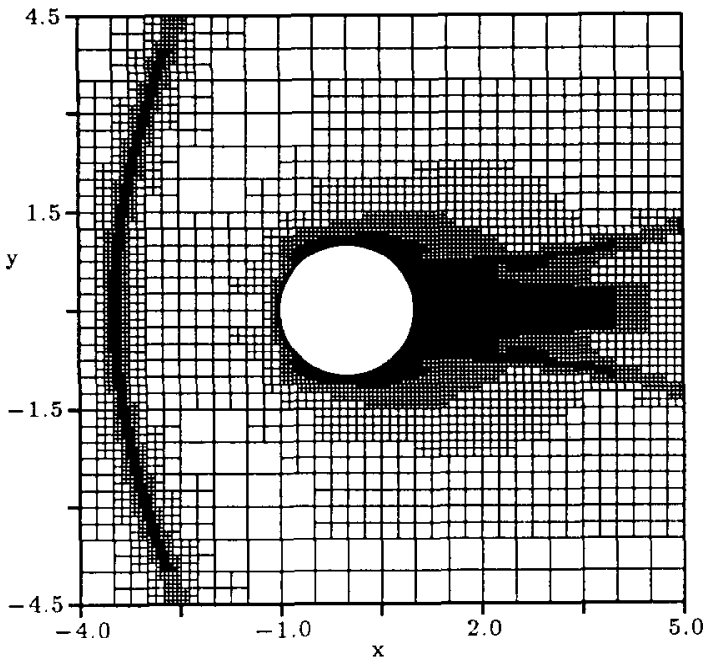

Fig. 26. Grid for conducting cylinder.

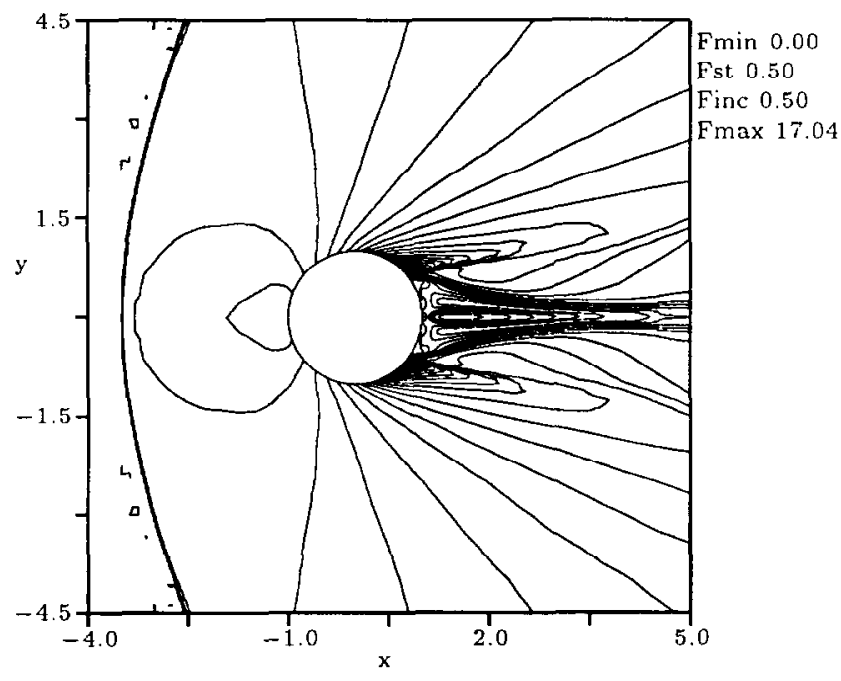

Fig. 27. Contours of Alfvénic Mach number for conducting cylinder.

relatively simple, shows the ability of the magnetohydrodynamic Riemann problem to capture weak solutions, and the power of incorporating this Riemann solver into the adaptive framework developed for the gas dynamics solver.

\section{Concluding remarks}

A method for solution of the equations of ideal gas dynamics and magnetohydrodynamics has been presented. Results for specific test cases show the power and flexibility of the scheme, specifically its ability to capture complex geometries and complex flow features automatically. The data structure is the crucial piece of the mesh-generation scheme, and the positive-reconstruction algorithm in combination with the upwind flux functions make the scheme work on the resulting meshes.

\section{Acknowledgments}

The calculations for the branched duct flow were carried out by Bill Coirier, a research scientist at NASA Lewis, and a doctoral student of the author. The blast wave calculations were carried out by Yu-Liang Chiang, a recently graduated doctoral student from the CFD group. Funding for the work presented here was provided in part by the National Science Foundation (Dr. George Lea), McDonnell Aircraft (Dr. August Verhoff), and NASA Langley (Dr. Jim Thomas). The author gratefully acknowledges all of their help. 


\section{References}

[1] T.J. Barth, On unstructured grids and solvers, in: Computational Fluid Dynamics, Von Kármán Institute for Fluid Dynamics, Lecture Series 1990-04 (1990).

[2] T.J. Barth and P.O. Frederickson, Higher order solution of the Euler equations on unstructured grids using quadratic reconstruction, AIAA Paper 90-0013 (1990).

[3] M.J. Berger and R.J. LeVeque, An adaptive Cartesian mesh algorithm for the Euler equations in arbitrary geometries, in: Proceedings ALAA 9th Computational Fluid Dynamics Conference (1989).

[4] M. Brio and C.C. Wu, An upwind differencing scheme for the equations of ideal magnetohydrodynamics, $J$. Comput. Phys. 75 (1988).

[5] Y.-L. Chiang, Simulation of unsteady inviscid flow on an adaptively refined Cartesian grid, Ph.D. Thesis, University of Michigan, Ann Arbor, MI (1991).

[6] Y.-L. Chiang, B. van Leer and K.G. Powell, Simulation of unsteady inviscid flow on an adaptively refined Cartesian grid, AIAA Paper 92-0443 (1992).

[7] J.F. Dannenhoffer III, Grid adaptation for complex two-dimensional transonic flows, Sc.D. Thesis, Massachusetts Institute of Technology, Cambridge, MA (1987).

[8] D.L. De Zeeuw and K.G. Powell, An adaptively-refined Carterian mesh solver for the Euler equations, $J$. Comput. Phys. 104 (1) (1993) 56-68.

[9] H. Paillère, K.G. Powell and D.L. De Zeeuw, A wave-model-based refinement criterion for adaptive-grid computation of compressible flows, AIAA Paper 92-0322 (1992).

[10] K.G. Powell, Vortical Solutions of the Conical Euler Equations, Notes on Numerical Fluid Mechanics 28 (Vieweg, Braunschweig, 1990).

[11] K.G. Powell, E.M. Murman, E.S. Perez and J.R. Baron, Total pressure loss in vortical solutions of the conical Euler equations, $A L A A J .25$ (1987).

[12] P.L. Roe, Approximate Riemann solvers, parameter vectors and difference schemcs, J. Comput. Phys. 43 (1981).

[13] P.L. Roe, Characteristic-based schemes for the Euler equations, Ann. Rev. Fluid Mech. 18 (1986) 337-365.

[14] C.-H. Tai, Acceleration techniques for explicit Euler codes, Ph.D. Thesis, University of Michigan, Ann Arbor, MI (1990).

[15] B. van Leer, Towards the ultimate conservative difference scheme, V: a second-order sequel to Godunov's method, J. Comput. Phys. 32 (1979).

[16] B. van Leer, W.T. Lee and K.G. Powell, Sonic-point capturing, in: Proceedings AIAA 9th Computational Fluid Dynamics Conference (1989).

[17] B. van Leer, C.H. Tai and K.G. Powell, Design of optimally-smoothing multistage schemes for the Euler equations, in: ALAA 9th Computational Fluid Dynamics Conference (1989).

[18] G. Warren, W.K. Anderson, J. Thomas and S. Krist, Grid convergence for adaptive methods, in: Proceedings ALAA 10th Computational Fluid Dynamics Conference (1991).

[19] S. Zachary and P. Colella, A second-order Godunov method for magnetohydrodynamics, J. Comput. Phys. (1992). 